\title{
FUSE Results on Magnetic CVs: VV Pup, YY Dra, LS Peg and DW UMa
}

\author{
Paula Szkody, Donald W. Hoard, Albert P. Linnell \\ Dept. of Astronomy, University of Washington, Box 351580, Seattle, \\ WA 98195 \\ Knox Long \\ Space Telescope Science Insititute, 3700 San Martin Drive, Baltimore, \\ $M D 21218$
}

Gary Schmidt

Steward Observatory, University of Arizona, Tucson, AZ 85721

Edward M. Sion

Department of Astronomy, Villanova University. Villanova, PA 19085

\begin{abstract}
In Cycles 2 and 3, we obtained FUSE observations of the Polar VV Pup, and the IP/SW Sex stars YY Dra, LS Peg and DW UMa. The spectra show a wide range of line intensities and structure. The phase-resolved spectra in each case reveal interesting properties about the hot accretion zones.
\end{abstract}

\section{Introduction}

FUSE provides important far-UV spectra that leads to new information about the hot accretion poles and the accretion curtains in magnetic CVs. The FUSE bandpass of $900-3000 \AA$ contains a wide range of line transitions over a large range of temperatures. The high resolution resolves narrow interstellar lines as well as narrow components within the broad emission lines from the accretion areas, and obtain velocities of the line-forming regions. Thus, the FUSE spectra can constrain the temperature structure in the accretion areas and obtain the geometry of the flow in magnetic systems. Some limitations to FUSE data are imposed by the low earth orbit i.e. gaps in the coverage due to earth blockage and significant airglow emission lines from oxygen and hydrogen. In addition, molecular hydrogen can be a problem for galactic plane locations. The current sensitivity reaches $\sim V<17.5$. We report results on one polar (VV Pup), one intermediate polar (YY Dra) and two SW Sex stars (LS Peg and DW UMa). 


\section{VV Pup}

This Polar has a $100 \mathrm{~min}$ orbital period, a distance of $145 \mathrm{pc}$ and a magnetic field strength of $\sim 30 \mathrm{MG}$ at the accreting pole. The geometry (i $75^{\circ}, \beta \sim 150^{\circ}$ ) results in the accretion pole being visible for about $45 \%$ of the orbit. The 11 orbits with FUSE (with simultaneous optical photometry) cover phases 0.7 to slightly past 0.0 . The phase-resolved FUSE spectra reveal an increasing continuum and decreasing line flux as the accretion spot comes into view. A hot $90,000 \mathrm{~K}$ black body fits both the FUSE and IUE fluxes, and gives a radius of $425 \mathrm{~km}$ for the FUV emitting area (details in Hoard et al. 2002).

\section{YY Dra}

YY Dra is an Intermediate Polar with an orbital period near $4 \mathrm{hrs,} \mathrm{a} \mathrm{white} \mathrm{dwarf}$ spin period of $529 \mathrm{sec}$ and a distance of $155 \mathrm{pc}$. The 2 contributing accretion poles lie close to the equator. The 14 orbits of FUSE spectra were phased on the spin cycle and show that the lines are $180^{\circ}$ out of phase with the continuum. BINSYN was used to model the phase resolved spectra with 2 spots of $200,000 \mathrm{~K}$ that have an angular radius of $5^{\circ}$ on a $21,500 \mathrm{~K}$ white dwarf.

\section{DW UMa}

This well-known eclipsing SW Sex star (IP?) has a high inclination $\left(82^{\circ}\right)$ and an orbital period of $3.3 \mathrm{hrs}$. The 6 FUSE orbits covered all phases except for phases 0.0 and 0.5. A narrow component feature that shifts from red (phase 0.9 ) to blue (phase 0.4) is evident in the CIII line and could be due to different views of the accretion stream or the magnetic accretion curtains.

\section{LS Peg}

This low inclination SW Sex star is one of only a few with measured circular polarization (Rodriguez-Gil et al. 2001). Unlike all the systems above, the lines of LS Peg are all in absorption, signifying a very thick accretion disk. Unfortunately, the contamination by molecular hydrogen is very strong, making it difficult to determine a proper continuum level. There is orbital variability in the OVI and CIII lines that may be related to the phase-related absorption effects in the Balmer lines that identify the SW Sex category.

This work was supported by NASA FUSE grants NAG 5-10343, 10246, 12203.

\section{References}

Hoard, D. W., Szkody, P., Ishioka. R., Ferrario, L., Gänsicke, B. T., Schmidt. G. D., Kato, T., \& Uemura, M. 2002, AJ, 124, 2238

Rodriguez-Gil, P., Casares, J., Martinez-Pais, I. G., Hakala, P., \& Steeghs, D. 2001, ApJ, 548, 49L 